\title{
Title: The Creation Process Of The First Particle From The Absolute Vacuum
}

\author{
Shivan Sirdy ${ }^{*}$ (Corresponding author) \\ Kirkuk university, Kirkuk, Iraq \\ *Corresponding Author Email address: sheevan91@gmail.com
}

\begin{abstract}
In this study, we discuss the properties of absolute vacuum space and how these properties can play a vital role in creating a mechanism in which the very first particle gets created simultaneously everywhere and we find the limit in which when the absolute vacuum volume reaches will lead to the collapse that leads to the creation of the first particle. This discussion is made following to the elementary dimensions theory study that was peer-reviewed at the end of 2020, everything in the universe is made from four elementary dimensions, these dimensions are the three spatial dimensions ( $X, Y$, and $Z$ ) and the Vacuum resistant as the factor of change among the four, time itself wasn't considered as the fourth dimension, rather time corresponds to a factor of change, and during the research it was found out that the Vacuum resistant is the factor of change in the Absolute Vacuum space, where time is a hypothetical concept, that represents changes during certain events compared to a constant change rate event.

Therefore, time does exist, but as a factor of change, and as the Vacuum resistant in the absolute vacuum space, Time $=$ factor of change $=$ Vacuum resistant.

In the study, the internal and external vacuum resistant volume equivalent is found, External Vacuum resistant $=3.2857602 * 10^{\wedge} 15 *$ mass. This equation is used to identify to amount of Free external vacuum resistant created during nuclear fission and fusion:

Initial mass of the excited nucleuses $\rightarrow$ mass of the created new nucleuses $+3.2857602 * 10^{\wedge} 15^{*}$ the lost Mass. In elementary dimensions the energy created during nuclear reactions is equivalent to the free External vacuum resistant created through nuclear reactions, and mass is equivalent to the internal Vacuum resistant.
\end{abstract}

Keywords: elementary dimensions; absolute vacuum; time alternative; early universe; universe at zero second; Vacuum resistant; Hydrogen atom; Hadron field; Lepton field

\section{Introduction}

How did the universe originate? What is beyond our great universe that we know today? what was before this universe?. All these questions are still under debate, and number of theories presented to draw a conceivable conclusion for the quest to get a right answer. The well-known theory is Big Bang, which predicts that the universe began from infinitesimal period of time from a point to a swelled balloon (the universe we know today) during a period of inflation [1]. The early phase of the 
universe was consisted of energy and very hot matter. The energy was consumed by some of the matter which congealed into unstable particles and with passage of time when cooled become stable particles. It is the similar concept proved through experimentations of nuclear fission and fusion reactions for making new stable and unstable nuclei by consuming intense amount of heat. The abundantly formed particles in early universe were thought to be hydrogen $(\mathrm{H})$ and helium $(\mathrm{He})$. This was later confirmed by many astrologists by measuring contents of $\mathrm{H}$ and $\mathrm{He}$ in galaxies, and stars. Later on, the gradual cooling of the universe results the formation of other elements which were forged in galaxies and stars. All aforementioned points of this model confirm its validity through experiments and observations including space-time. But still there are many discrepancies in the model which further need improvements by valid theory. For example, the time frame of rapid inflation is not directly tested, and what sort of energy provoked this inflation. Inflation is an extremely powerful theory in support to early events responsible for big bang, but unfortunately this theory doesn't explains the cause of inflation [2].

In recent years, another theory is proposed as an alternate to Big Bang model i.e. "bouncing cosmology" [3]. According to this theory, the universe was born multiple times periodically in endless cycles of continuous expansion and contraction. The concept of periodic expansion and contraction provide support to interconnect big bang and inflation theory very effectively. While some points are still controversial in theory of bouncing cosmology like first origin and how many cycles the universe has completed till now. One of the fundamental pillars of the theory still required a valid answer which could explain about the self-renewing of the universe. Gamow et al. [4] suggested that early universe immediately transformed from instability to stability which results the formation of fundamental and heavy particles due to fusion energy. This idea reflects projection in our modern experimentations which validates the idea and rapidly endorsed by the big bang theory.

The commonly agreed upon key points by all theories are as follows.

1. The universe expands its cools in comparison to its early form.

2. In the very beginning of big bang, when universe was intensively hot, it exhibits a lot of 
confined radiation energy and matter coexisted.

3. Big bang is the result of forming of matter and antimatter

Both points have been endorsed by all theories to date. Specially the second point, in which experimentations are performed to identify the exotic states of matter in early universe.

The third point need further attention which is not been discussed previously that for how long the matter and anti-matter co-existed.

The goal of the paper is to provide an alternate answer to the questions about the origin of the universe

The scientific contributions of the paper are:

1. Introduce the Vacuum resistant as a replacement to a factor of change, and as a Time in the absolute vacuum space.

Time $=$ factor of change $=$ Vacuum resistant

2. Present the limit of the level of instability in the Absolute Vacuum space.

3. Provide an alternate solution to the origin of matter.

4. Introduce the hadron and lepton field as internal and external Vacuum resistants

5. Introduce External vacuum resistant equation: External Vacuum 


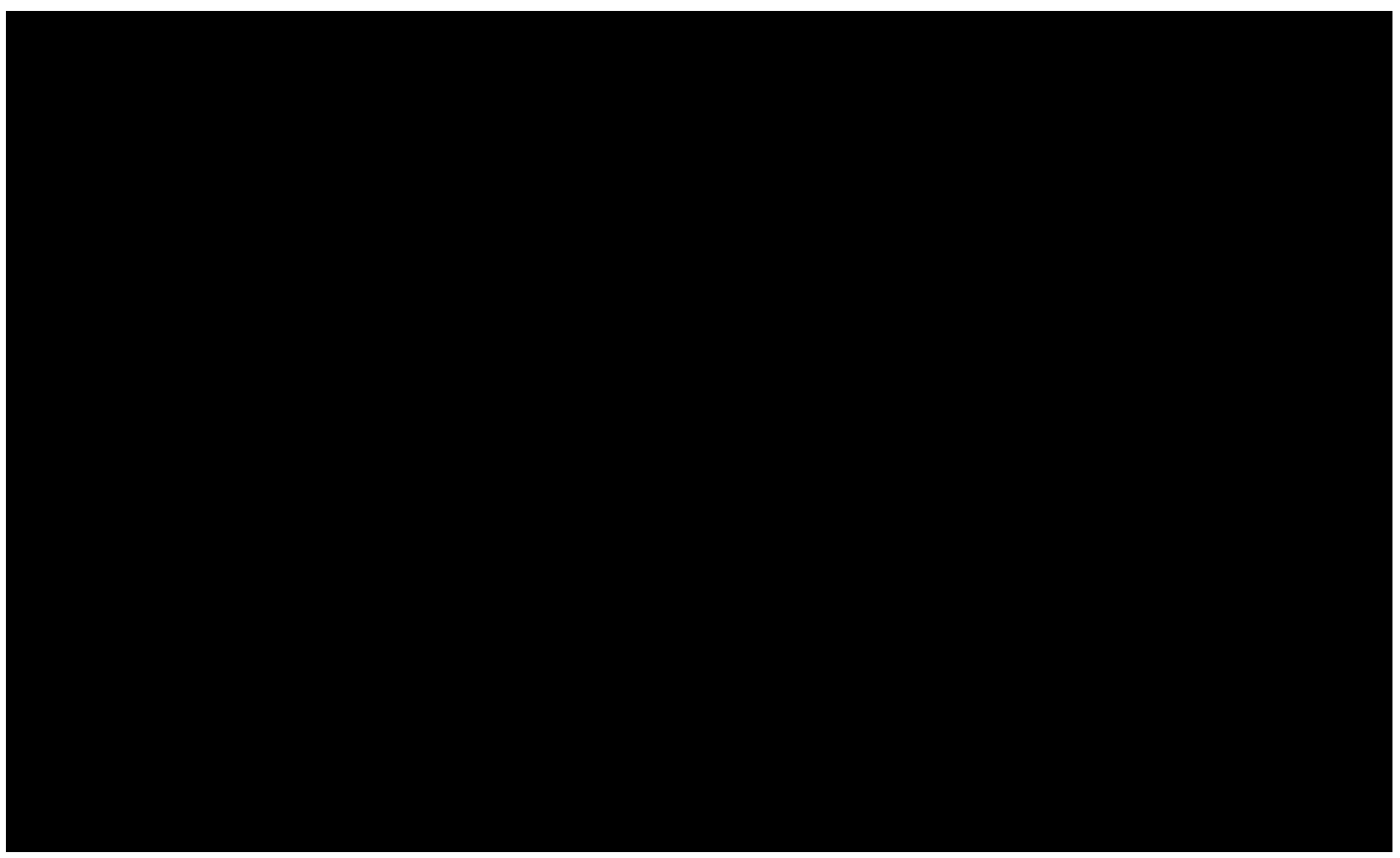

Figure 1(universe at time $=0$ sec or absolute vacuum)

There are two major foundations for this study that are in same time opposing the current physics literature:

1. Time corresponds to a factor of change. Instead of space-time terminology, we have space-change in this study. The only difference between now and 15 billion years ago is the fact that changes happened and not that time has passed.

2. Universe at zero second (shown in figure 1) was naturally at Absolute zero temperature. Absolute zero temperature can be achieved theoretically by extracting all sorts of particles and radiations. Universe at zero second was at Absolute zero temperature because it was completely devoid of everything [6]. Hence, Universe at zero second was Absolute vacuum.

Creating absolute vacuum in the laboratory is next to impossible if not impossible itself due to difficulty of the removal of all sorts of matter and radiation for the creation of absolute zero temperature.

Absolute vacuum is four-dimensional (space dimensions in the $x, y$, and $z$ axis and the Vacuum resistant). The Vacuum resistant is the factor of change among the four dimensions [6] which plays 
a vital factor in creating the first particle. Those four are the elementary dimensions. In the previous study the Vacuum resistant was named Force field, however since it was creating confusion with the force known in physics it was changed to Vacuum resistant.

Time is a hypothetical concept [5], that corresponds to changes during certain events compared to a constant change rate event [6]. Therefore, time itself wasn't considered as the fourth dimension. Though, time does exist but as a factor of change, and the Vacuum resistant was identified as a factor of change in the absolute vacuum space as it will be explained below.

Time $=$ factor of change $=$ Vacuum resistant

Therefore, every function that will be used in the EDs (the elementary dimensions) theory, are time invariant function [7].

This paper includes:

- Identification of the fourth dimension experiment: in his part we discuss the methodology that was used to identify the fourth dimension.

- Vacuum Resistant patterns: In this part we discuss the patterns of the Vacuum Resistant.

- Creation of the first particle through the elementary dimensions and the limit of the collapse: In this part we discuss the methodology where the first particle gets created simultaneously everywhere in the space of Absolute Vacuum, then we find the limit where the system collapses creating the first particle, then we find the external and internal Vacuum resistant equivalent that governs the Radius of the lepton field in relation to the hadron field.

- The collapse of a neutron to a proton + Lepton: In this part, we discuss the process in which a neutron collapses to a proton and a lepton.

- Nuclear fusion and fission: in this section, we introduce the nuclear reactions using the internal and external vacuum resistants.

\section{Methodology}

\section{Identification of the fourth dimension}

The Vacuum resistant or the factor of change was identified through experiment, Owing to lack of resources, a $10 \mathrm{ml}$ syringe was used to identify the fourth elementary dimensions of the vacuum. The syringe head was closed, and the bottom part was pulled by using weights to create the vacuum in the syringe. (Shown in figure 2) 


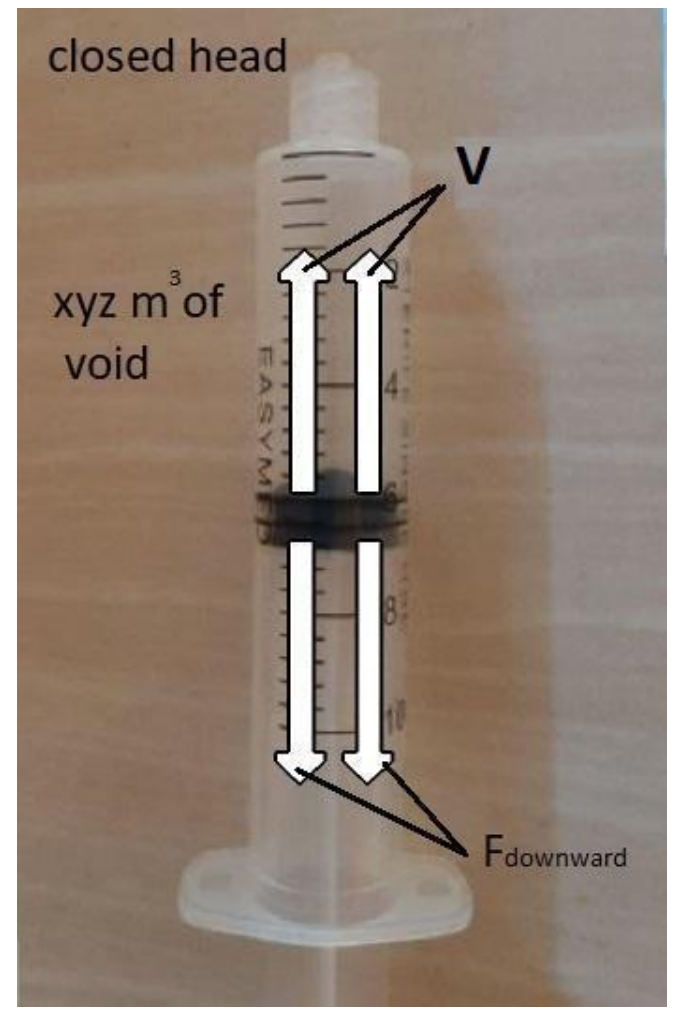

Figure 2 identification of the fourth dimension

The resistant force developed inside the syringe due to lack of matter was called Vacuum resistant or just $\mathrm{V}$ for simplifications

Through classical physics, the summation of forces on the vertical axes equals zero

$\Sigma$ Fy=0, V+ Fdownward $=0$.

Where Fdownward is the force needed to pull the matter downward to create xyz $\mathrm{m} 3$ of vacuum, and $V$ is the vacuum resistant sourced from the vacuum to resist the lack of matter.

$\mathrm{V}+$ Fdownward $=0, \mathrm{~V}=$-Fdownward.

Therefore, the $\mathrm{V}$ or the vacuum resistant corresponds to the factor of change

\section{Vacuum resistant patterns}

The figure 3 and 4 shows the Vacuum resistant pattern direction of an absolute vacuum in a confined and an open system, respectively. 


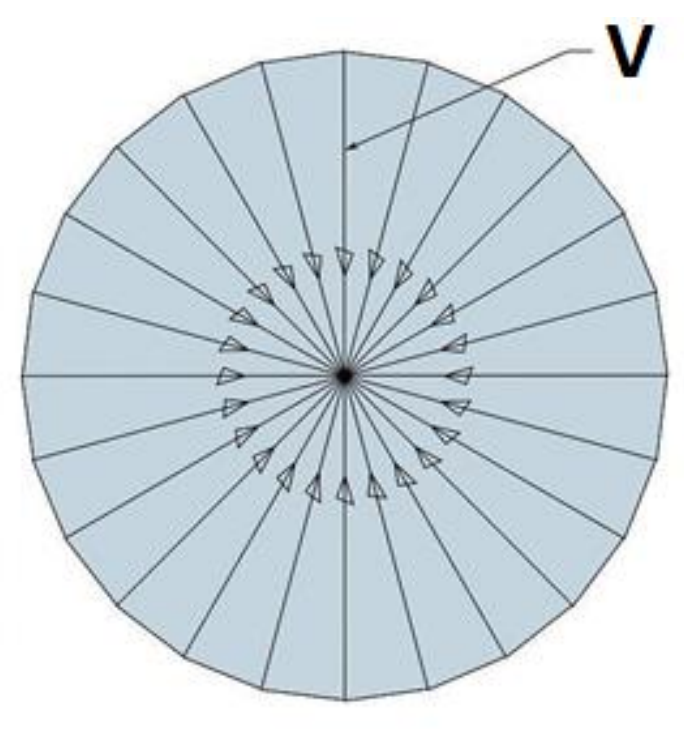

Figure 3 Vacuum resistant patterns in a confined system

In Figure 3 the Vacuum resistant acts to prevent the formation of the vacuum by trying to crush the parameter of the surroundings toward the centre; the direction of $V$ is pointing toward the centre of the vacuum. 


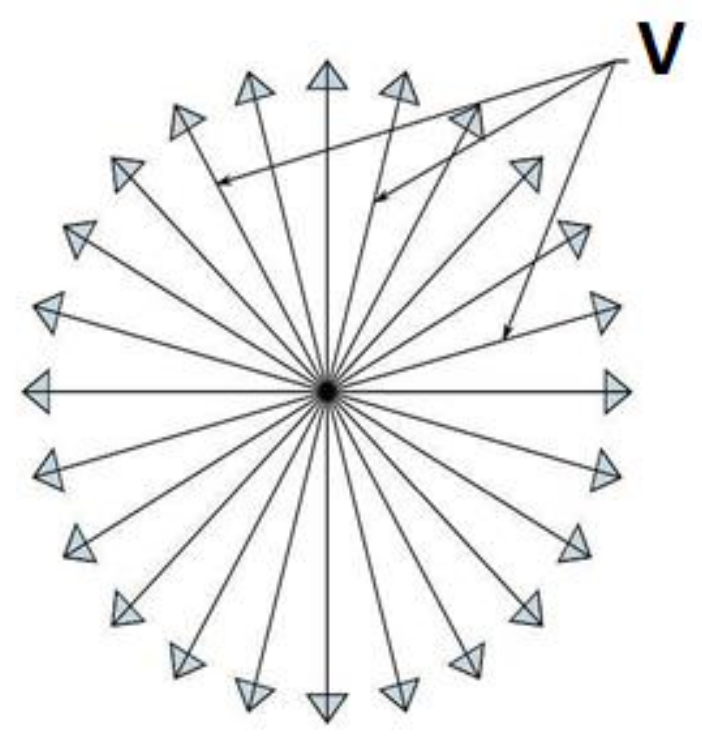

Figure 4 Vacuum resistant pattern in an open system

However, absolute vacuum is considered an open system, if it exists in a free form. At any point of it the Vacuum resistant is pointing outward, as depicted in Figure 4.

Any point of absolute in a confined system acts as absolute vacuum in an open system, which means that Vacuum resistant is pointing outwards (see Figure 5).
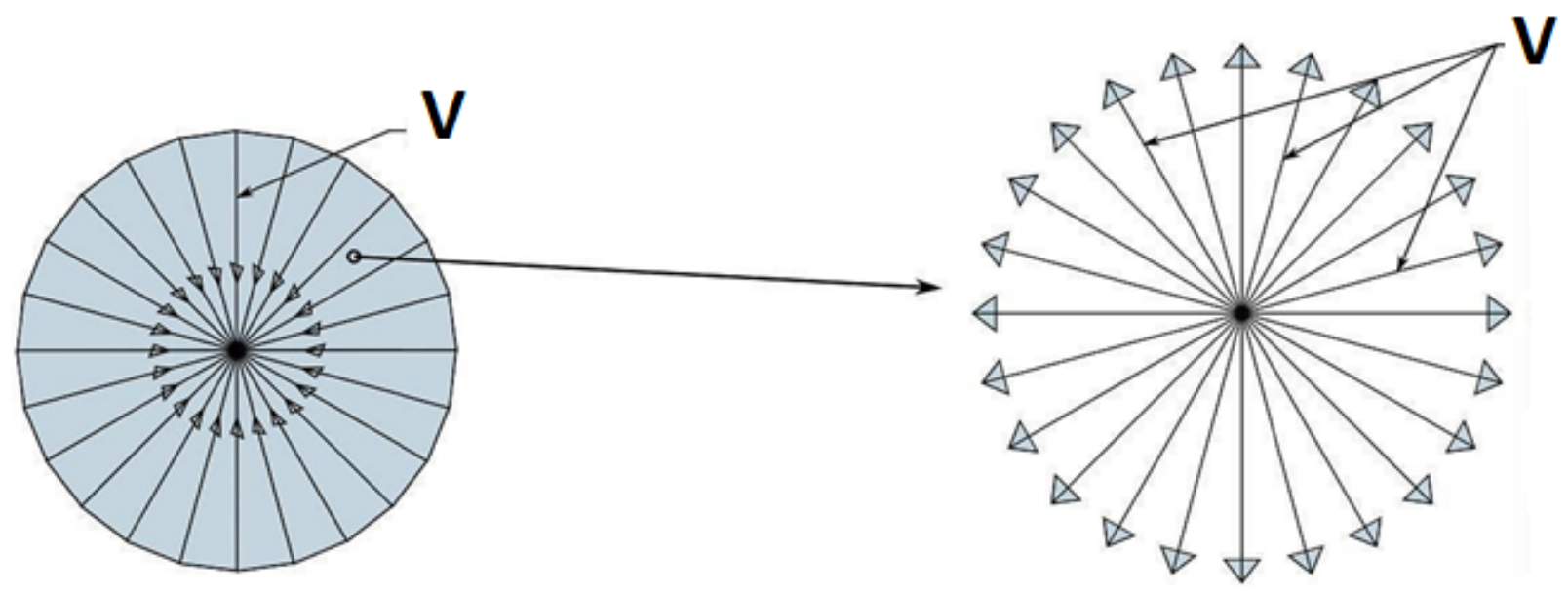

Figure 5 every point inside a confined system acts as an open system 
The Vacuum resistant $(\mathrm{V})$ has a positive relation with the spatial dimensions ( $\mathrm{X}, \mathrm{Y}$ and $\mathrm{Z}$ ), meaning the Vacuum resistant increases with the increase of the absolute vacuum's volume $V v$.

$\mathrm{V} \alpha \mathrm{Vv}$

$\mathrm{V}=\mathrm{Cv} * \mathrm{Vv}$

Where $\mathrm{C} v$ is the vacuum constant that was assumed, here we will again assume that it's equal to 1 with $\mathrm{SI}$ base units of $\mathrm{Kg} \cdot \mathrm{m}-2 \cdot \mathrm{sec}-2$

The volume of sphere $V v=\frac{4}{3} \pi(R 3)$

$V=\frac{4}{3} \pi(R 3) C V$

$V$ will have the units of force $\mathrm{N}$ (Newton), excluding the time effect completely, as this will be as well a time invariant function.

\section{Creation of the first particle through the elementary dimensions}

According to the elementary dimensions EDs theory, the absolute vacuum and its four EDs are the predecessor of particles [6], and the EDs makes up everything in the universe. Contrarily to the current theories, according to the EDs theory a dot particle (or a one dimensional particle) is impossible to exist due to the mathematical impossibility and the absence of an existing actual physical limit determining the smallest mathematical and physical size. Therefore, every particle no matter how tiny it's, it must be having the three spatial dimensions ( $X, Y$, and $Z$ ) and according to the EDs theory it also has the fourth dimension (the factor of change or the Vacuum resistant). 


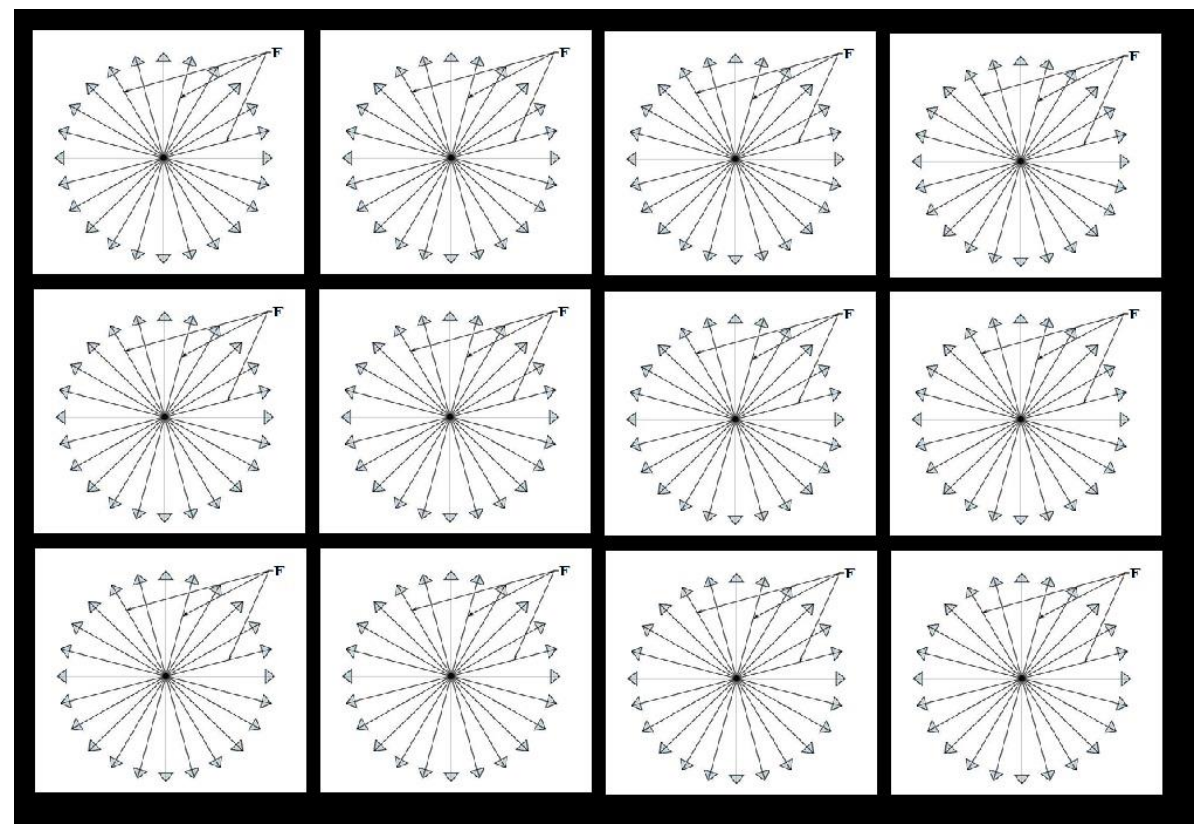

Figure 6 Universe at zero second with radius of $X$

Figure 6 visualizes a cross section of the universe at zero second and how it's only made of the outward Vacuum resistant, as the system is an open system.

With the increase of the absolute vacuum's volume, the outward Vacuum resistant increases, and the space losses equilibrium more and more due to the presence of only one directional Vacuum resistant. When the outward Vacuum resistant reaches a certain limit (the limit will be discussed below), the system reaches the highest level of instability. The outward Vacuum resistant has to collapse inward to restore equilibrium in the space (see Figure 7).
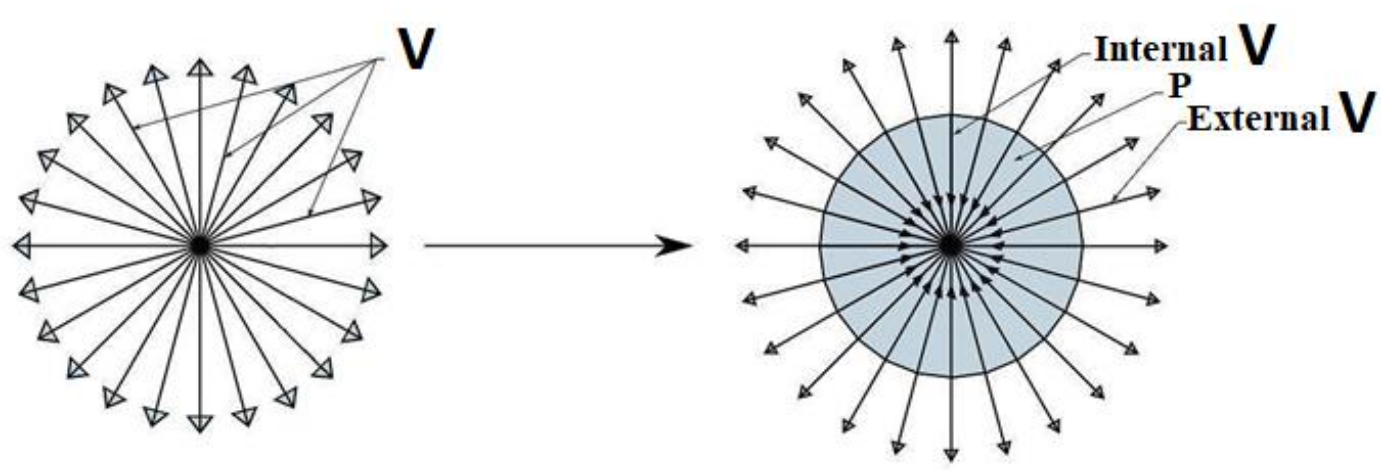

Figure 7 the change from an open system to a confined system 
The absolute vacuum has turned from an open to a confined system, with the collapse energy was created from the Vacuum resistant pointed toward the centre, the energy stays conserved at all phases. However, due to the collapse the energy density increases with the decrease of the spatial dimensions (see figure 8).

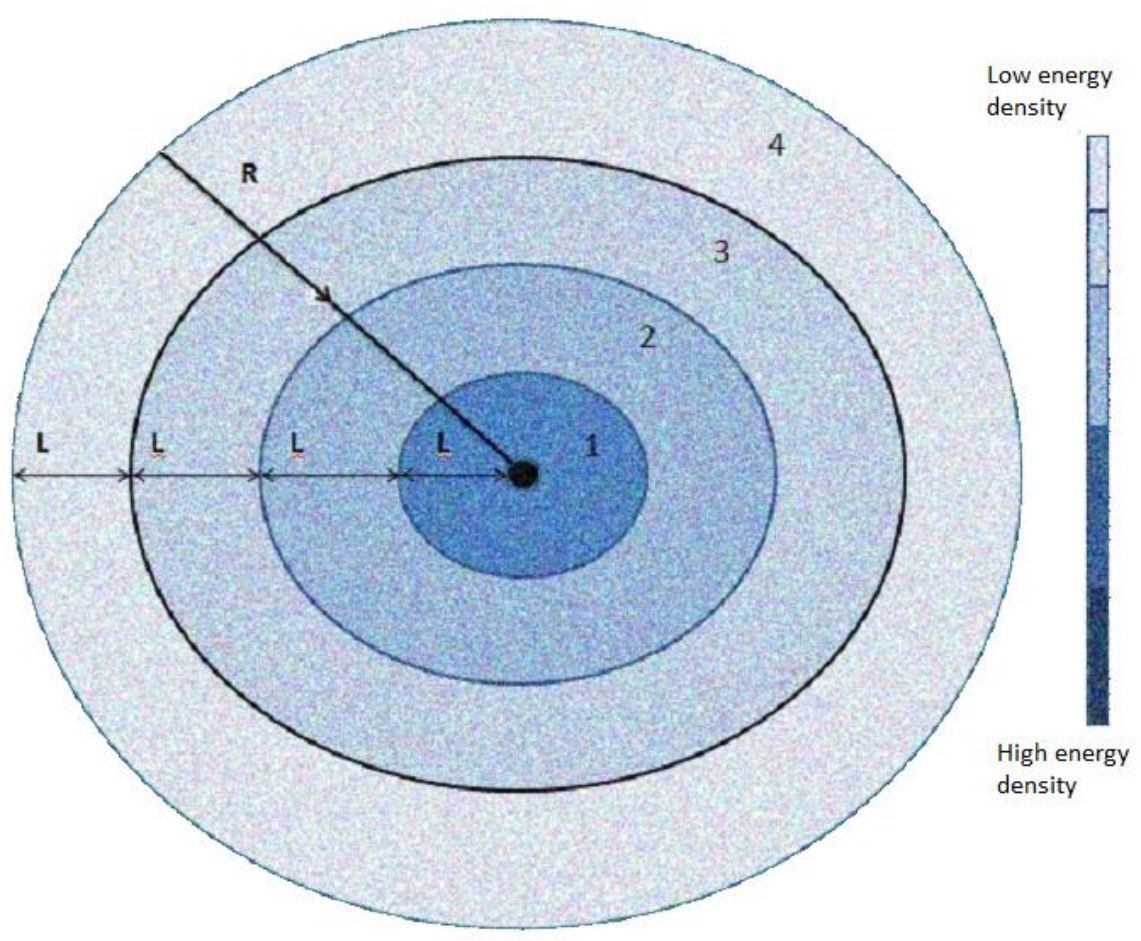

Figure 8 increase of the energy density with the collapse

At the final stage the energy is concentrated forming a solid shape of energy. In which, this shape of energy is a first particle that was ever created from the EDs. This particle was created to decrease the instability in the space to achieve equilibrium that the universe at time zero second was lacking.

This particle is managed by two forces:

1. Internal Vacuum resistant $\mathrm{Vi}$, which originates from the absolute vacuum inside the particle, just like vacuum in a confined system, and is directed toward the centre.

2. External Vacuum resistant Ve, originates from the absolute vacuum outside the created particle.

The Vacuum resistant Vi causes the source to collapse, while the Vacuum resistant Ve is the counter factor. The particle collapses until equilibrium is achieved between the internal and external Vacuum resistant. In case, more than one source-like entity are existents near each other relatively, the equilibrium process is among all of them. 
At time zero second, infinite spatial dimensions of absolute vacuum existed. Therefore, unlimited numbers of particles were created (see figure 9).

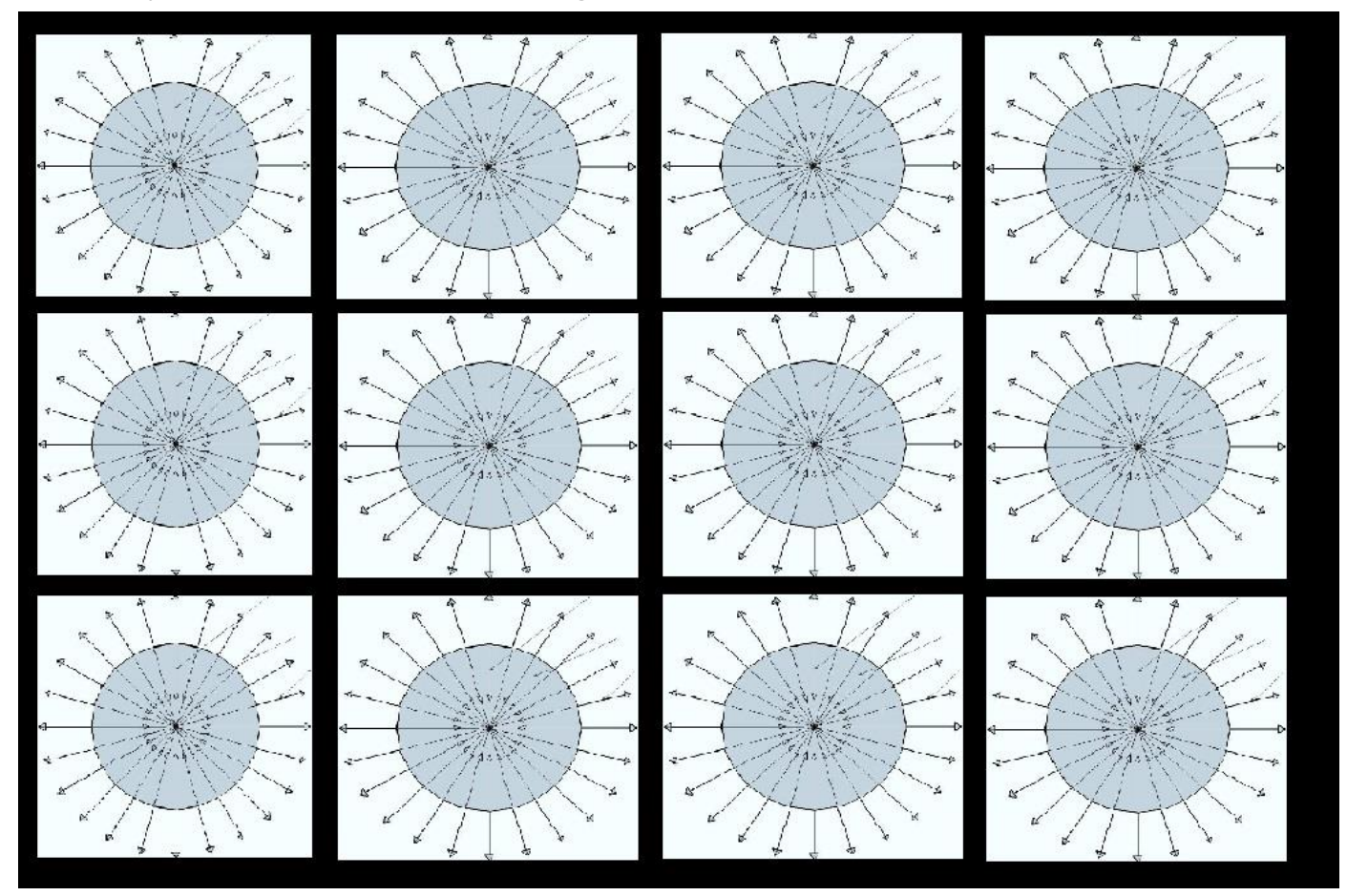

Figure 9 (infinity numbers of particles created from the EDs)

Figure 9 visualizes a cross section of the universe after the collapse and how the system has turned from an open system to a confined system.

The limit in which the system collapses might be the same limit that leads to the creation of the black holes, where it's already know that for large stars above the Landau/Tolman-OppenheimerVolkoff limit [8] (around two solar masses), when the star dies the forms of particles cannot provide the force needed to balance gravitational field. There is nothing to stop the collapse forming a black hole [8].

A black hole is similar to the origin particle or the source [6].

The limit could be a radius of $8.7713 \times 10^{8}$ meters of absolute vacuum in a sphere shape, using a value of $3.98 \times 10^{\wedge} 30 \mathrm{Kg}$ (two solar masses) with density of $1408(\mathrm{~kg} / \mathrm{m} 3)$, the same density of our 
sun.

Using mass density equation:

Denisity $=\frac{\text { two solar masses }}{\text { Volume of the star }}$

$1408=\frac{3.98 \times 10^{\wedge} 30 \mathrm{Kg}}{\frac{4}{3} \pi\left(\mathrm{R}^{\wedge} 3\right)}$

We get the value of a radius $8.7713 \times 108$ meters of absolute vacuum,

Using the Vacuum resistant equation (6):

$V=\frac{4}{3} \pi(R 3) C v$

$V=2.82671 \times 10^{\wedge} 27 \mathrm{~N}$

Meaning when the absolute vacuum's Vacuum resistant reaches this value, the space inside the sphere will be at its highest level of instability, the only way for it to achieve equilibrium is to collapse inward.

The spatial dimensions of the two solar masses its radius equivales to its Schwarzschild radius, Meaning the open system radius is equivalent to its closed system radius

Radius of two solar masses=Its Schwarzschild radius* equivalent.

$8.7713 \times 10^{\wedge} 8=5.9 * 10^{\wedge} 3^{*}$ Radius equivalent

Radius Equivalent=148666.1

Meaning:

Open system radius $=148666.1 *$ closed system radius

Open system is the external vacuum resistant 
Closed system is the internal vacuum resistant

Of course, the same thing can be said to their volume, which means that we also have Volume equivalent:

External vacuum resistant Volume= Internal vacuum resistant Volume $*$ Volume equivalent.....(10)

$4 / 3 * \mathrm{Pi}^{*}\left(8.7713 \times 10^{\wedge} 8\right)^{\wedge} 3=4 / 3^{*} \mathrm{Pi}^{*}\left(5.9^{*} 10^{\wedge} 3\right)^{\wedge} 3^{*}$ Volume equivalent

Volume equivalent $=3.2857602 * 10^{15}$

External Vacuum resistant $=3.2857602 * 10^{15} *$ Internal Vacuum resistant.

Hydrogen radius

Using radius equivalent

Atom radius $=148666.1 *$ Proton radius (12)

$=148666.1 * 0.85 * 10^{\wedge}-15=200.5422 * 10^{\wedge}-12$ meter $=126.3662$ picometer
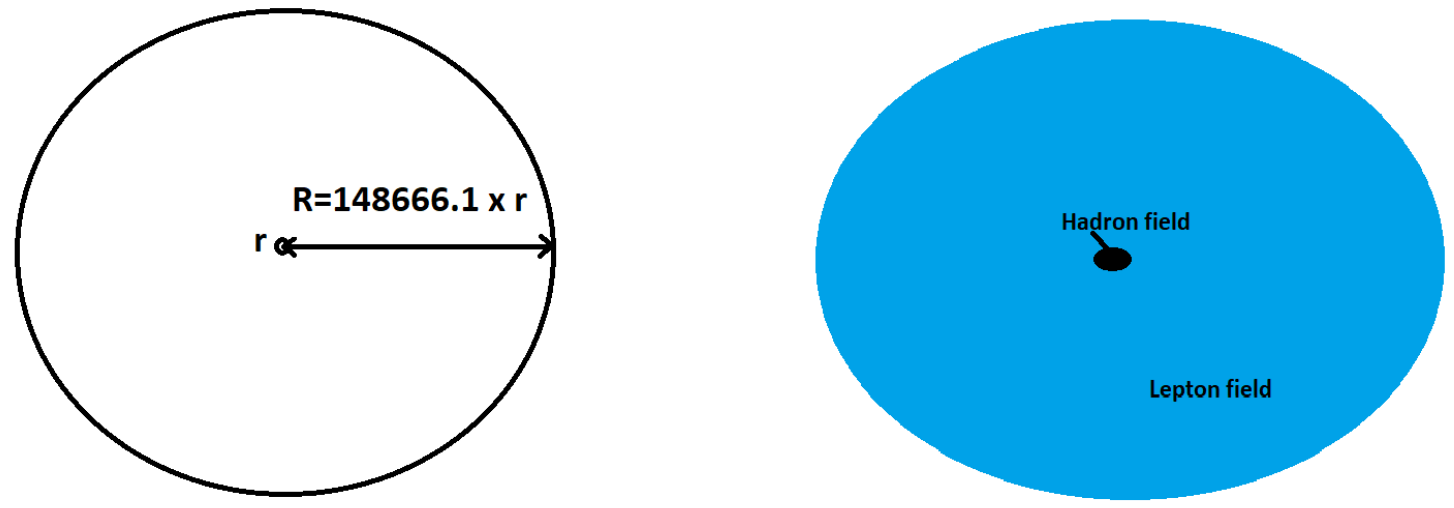

Figure 10 Hydrogen atom sample analysed using the external and internal Vacuum resistant ratios 
4. The collapse of a neutron to a proton + Lepton

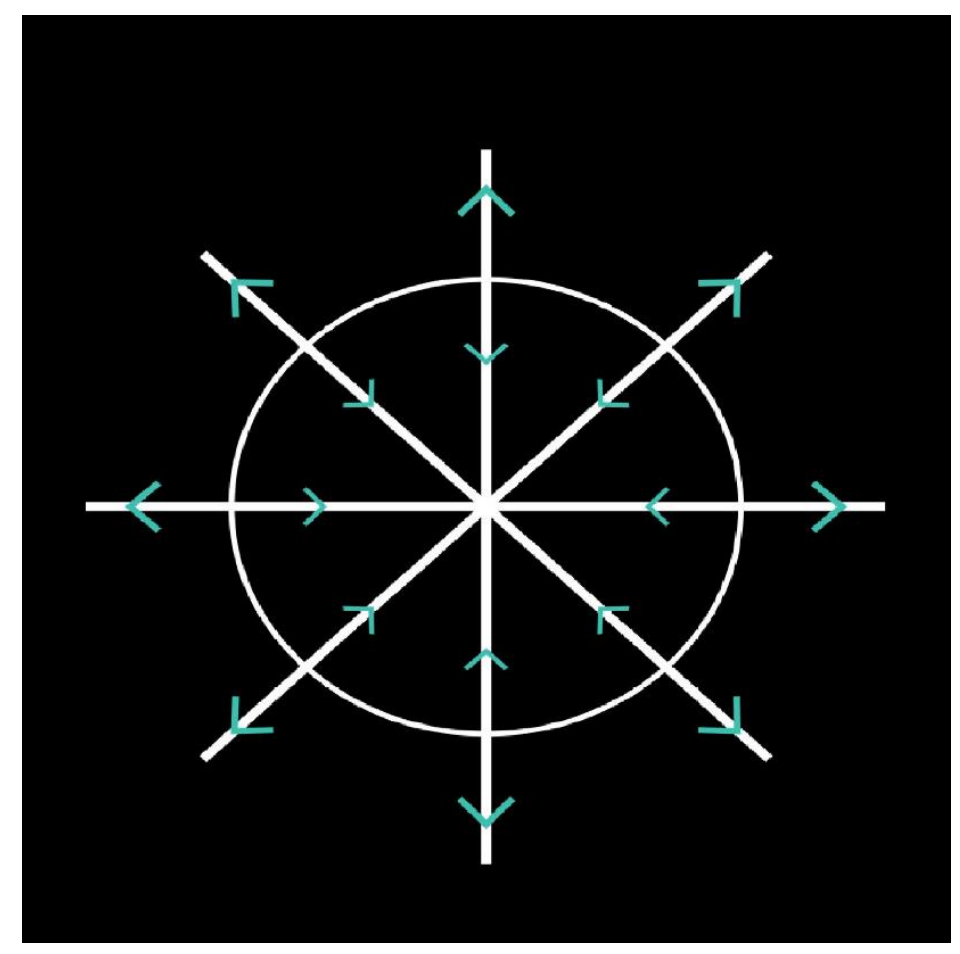

Figure 11 Neutron

The above Figure 11 shows a neutron before it collapses, as a neutron is a particle governed by the Internal Vacuum resistant that tends to make the particle collapsing and the External Vacuum resistant that tends to stop the collapse. In a neutron, the Internal Vacuum resistant is greater than the internal Vacuum resistant, therefore the external Vacuum resistant is not enough to stop the particle from collapsing. 


\section{Hydrogen atom}

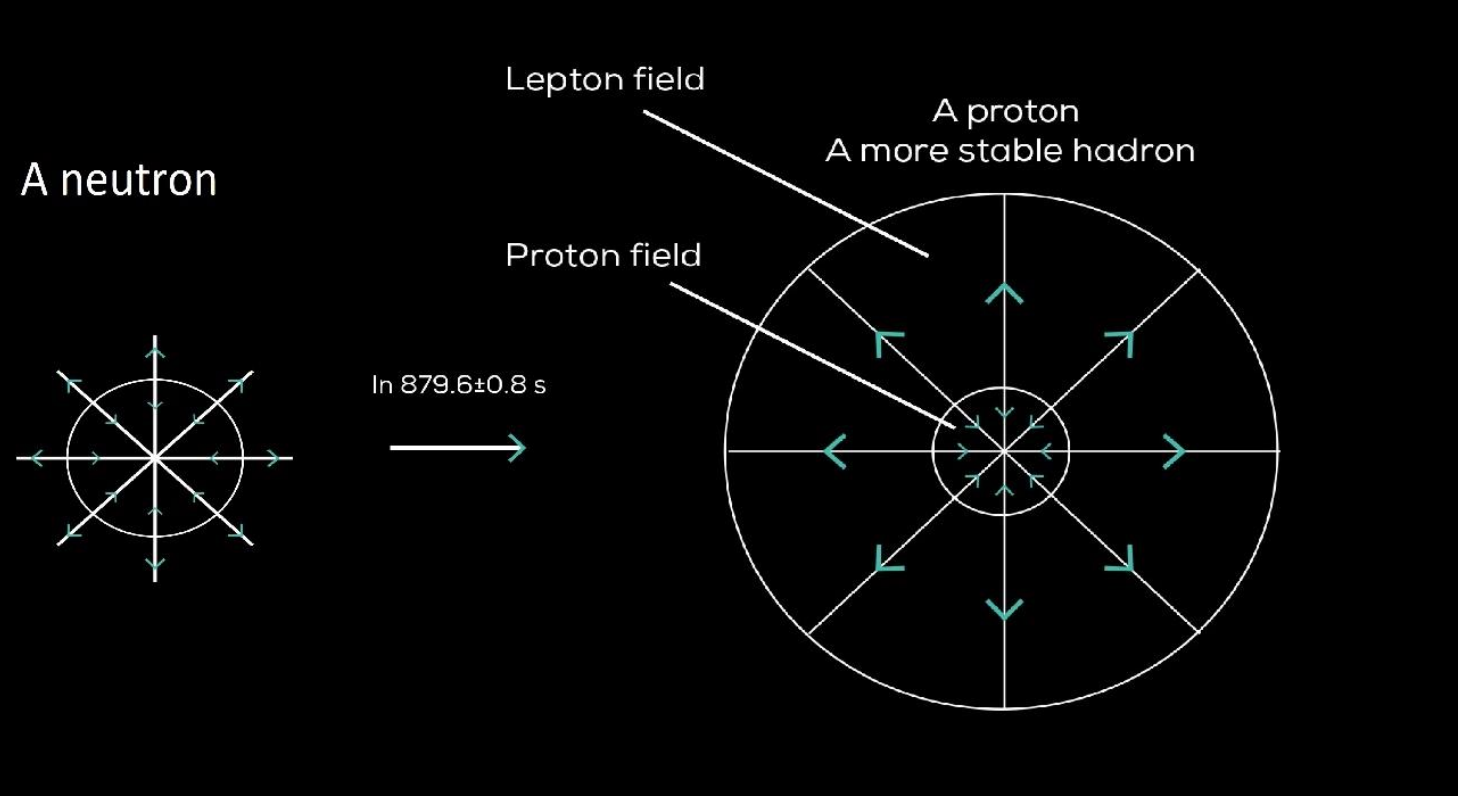

Figure 12 the collapse of a neutron to a proton+Lepton

In figure 12, in 879.6 plus minus 0.8 seconds a neutron has collapsed due to the effect of the Internal Vacuum resistant being larger than the External Vacuum resistant, and when both of the resistants are equal to each other, the system of the particle becomes in equilibrium, The Internal Vacuum resistant will be called a proton field, and the External Vacuum resistant will be called the Lepton field. Whenever, a system collapses, electro-magnetic field gets created from the effect of the External Vacuum resistant on the system as a free field spreading outward (see figure 13, 14, and 15).

As we can see above in the figures that the internal vacuum resistant is equivalent to mass Mass=Internal vacuum resistant. . ...

Returning the equation (11):

External Vacuum resistant $=3.2857602 * 1015 *$ Internal Vacuum resistant

(11)

The internal vacuum resistant can substituted with mass,

External Vacuum resistant $=3.2857602 * 10^{\wedge} 15 *$ Mass 


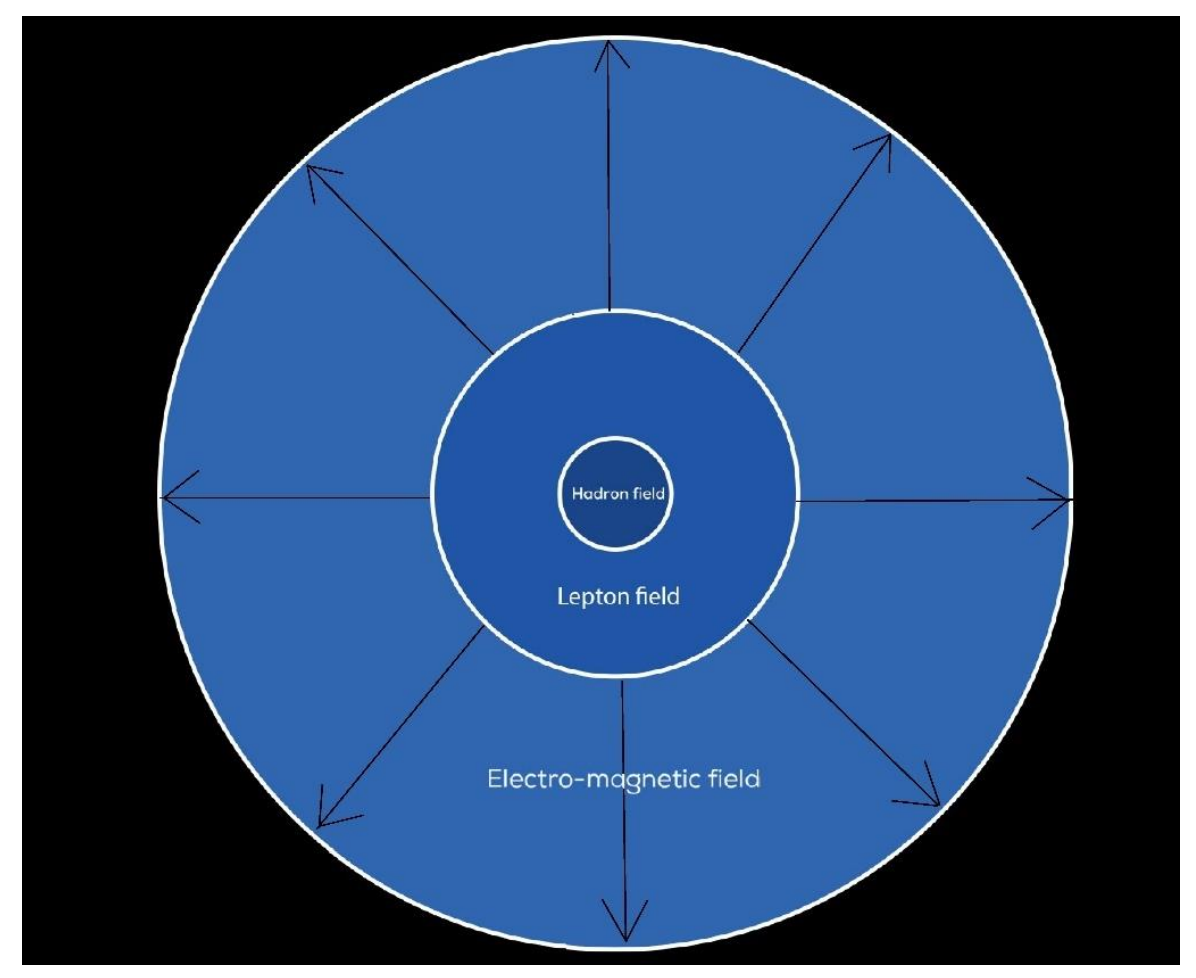

Figure 13 The three main fields

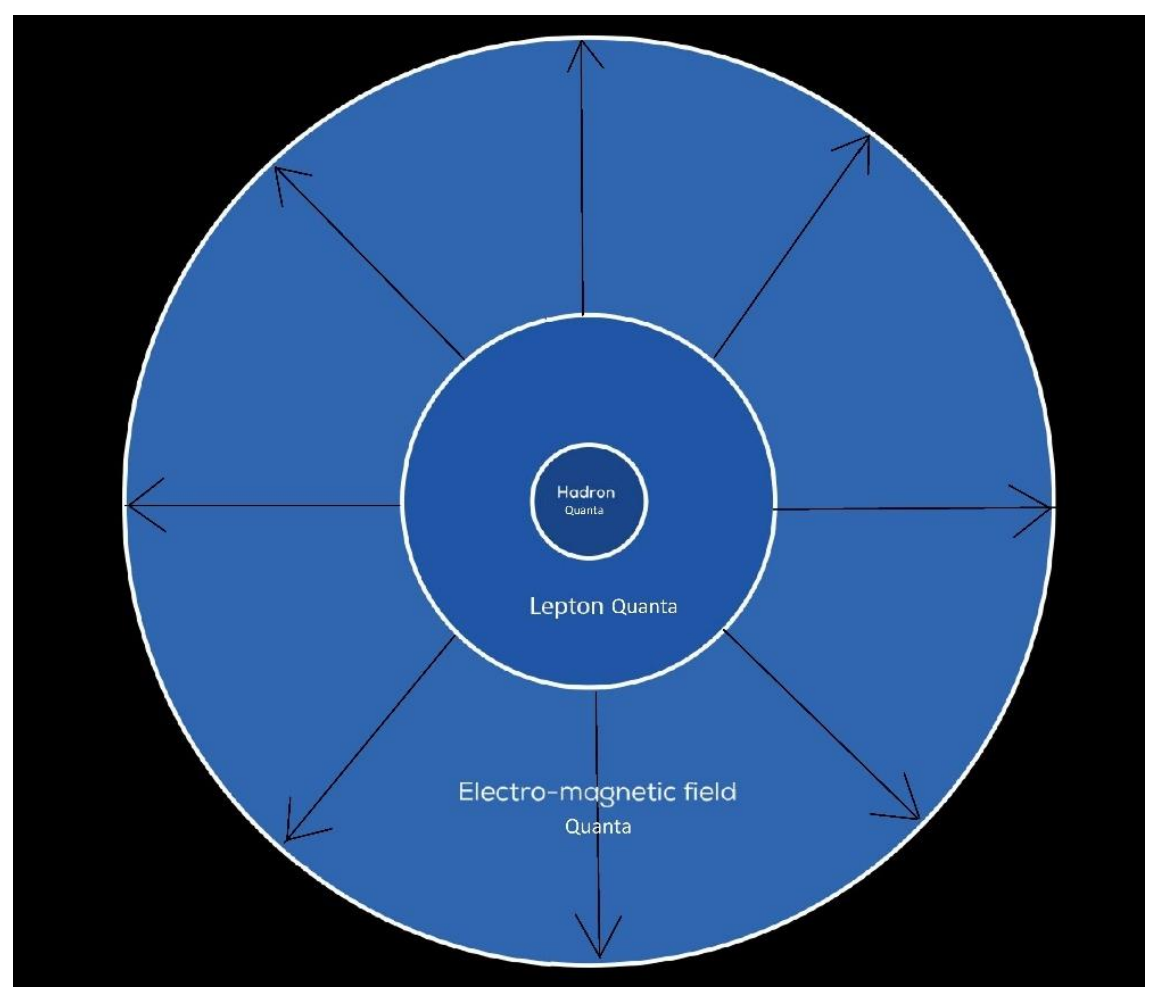

Figure 14 each of the three fields can be quantized 


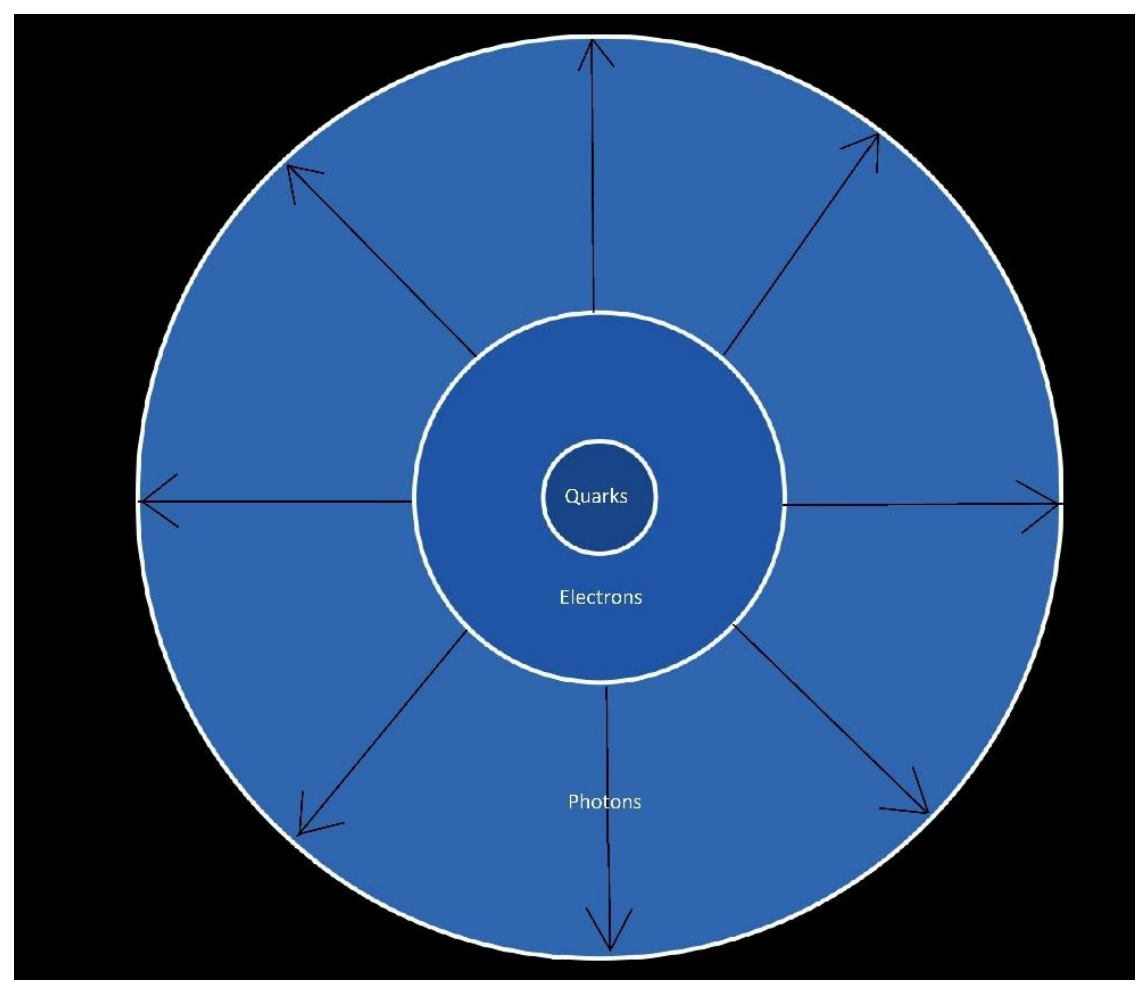

Figure 15 Quanta of each of the three main fields

\section{Nuclear fusion and fission}

Nuclear Fission is the process of splitting of a heavy, unstable nucleus into two lighter nuclei, and fusion is where two light nuclei combine releasing an amounts of energy [9], or something else.

It's known that the nuclear fusion and fission can be expressed as the below formula:

Initial mass of the excited nucleuses $\rightarrow$ Mass of the created new nucleuses + Energy

According to the EDs theory, mass is equivalent to the internal vacuum resistant, while energy is not defined yet. There is only one way for energy concept to fit in the EDs theory, and that is the fact that energy is the free external vacuum resistant,

Free External Vacuum resistant=Energy.

Now we return to formula (15), but this time we use the EDs fundamental properties:

Initial Internal Vacuum resistant of the excited nucleuses $\rightarrow$ Internal Vacuum resistant of the created new nucleuses + External Vacuum resistant

And since External vacuum resistant $=3.2857602 * 10^{\wedge} 15 *$ Mass (equation 14 ),

Initial Internal Vacuum resistant of the excited nucleuses $\rightarrow$ Internal Vacuum resistant of the created new nucleuses $+3.2857602 * 10^{\wedge} 15 *$ the lost Mass

We can put the above formula in a calculable shape through:

Initial mass of the excited nucleuses $\rightarrow$ mass of the created new nucleuses $+3.2857602 * 10^{\wedge} 15 *$ the lost Mass

$3.2857602 * 10^{\wedge} 15 *$ the lost mass represents the amount of the free external vacuum resistant created 
through the process of the nuclear fusion or fission.

Now through the above formula we are able to accurately calculate the amount of change that occurs in a system during nuclear fusion or fission.

Taking Deuterium- Deuterium fusion as an example:

${ }_{1}^{2} \mathrm{H}+{ }_{1}^{2} \mathrm{H} \rightarrow{ }_{2}^{3} \mathrm{He}+{ }_{0}^{1} n+3.27 \mathrm{MeV}$

${ }_{P}^{A} N$, where $\mathrm{N}$ is nucleus, A number of protons plus neutrons, $\mathrm{P}$ is number of protons, and $\mathrm{n}$ is neutron

Now shifting to the EDs principles:

${ }_{1}^{2} H+{ }_{1}^{2} H \rightarrow{ }_{2}^{3} \mathrm{He}+{ }_{0}^{1} n+$ Lost mass $* 3.2857602 * 10^{\wedge} 15$

And the same applies to every other fusion or fission.

\section{The relation to the General Relativity:}

The results in this article doesn't disagree with the General relativity, however, only the visualization of the space-time is different. Where instead of space-time, we have space-change here.

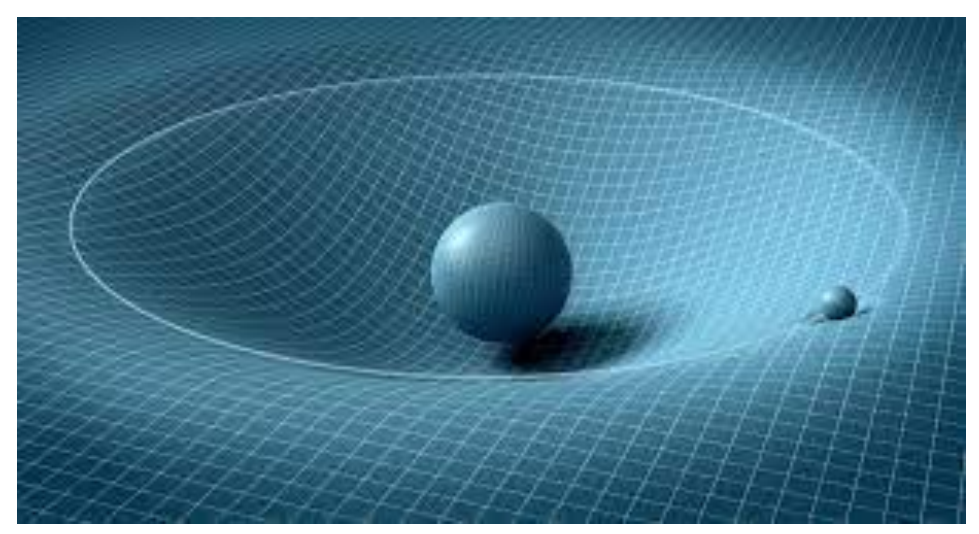

Figure 16: General Relativity's visualization on the gravitation around objects

Fig. 8 shows the General Relativity's visualization of the space-time and gravitation. Where the curvature is stronger getting nearer to the object.

While according to this article, everything that has been came up with in the General relativity are correct, except that the concept of space-time is replaced with space-change. As already stated that time corresponds to changes in a system compared to constant change rate event. 


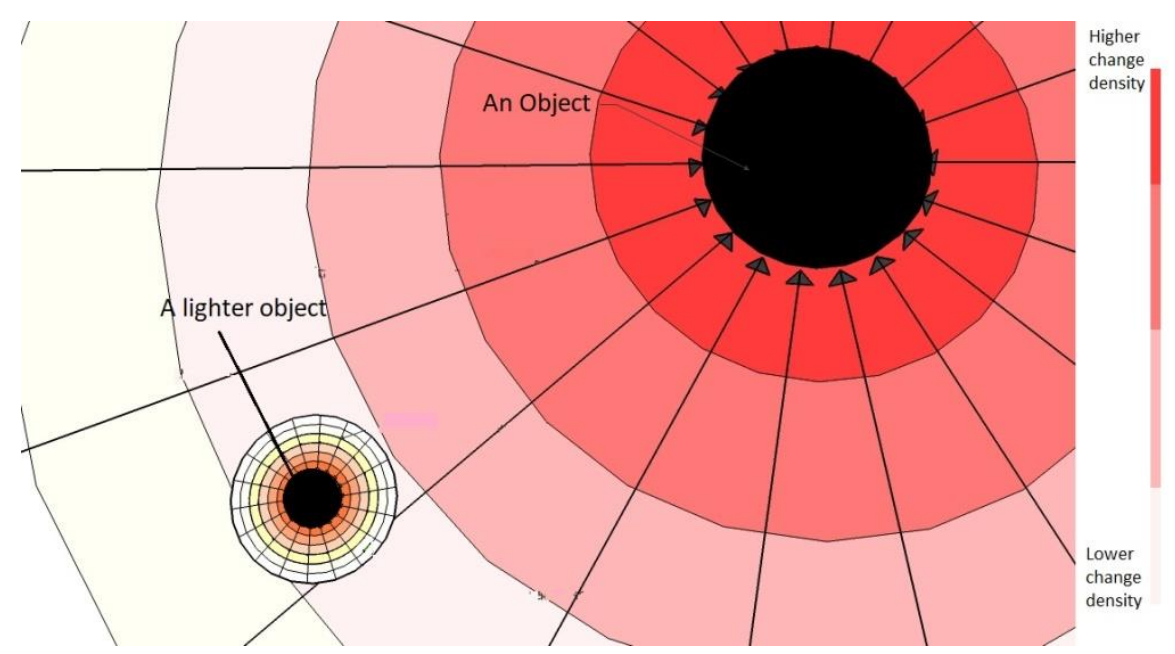

Figure 17: The Elementary dimensions theory's visualization on gravitation around objects

Fig. 9 shows instead of the curvature getting stronger near the object, the change density gets stronger.

\section{Conclusion}

In the universe at zero second only absolute vacuum existed, absolute vacuum is made from EDs., three spatial dimensions ( $X$ axis, $Y$ axis and $Z$ axis) and the Vacuum resistant. When the sphere volume of absolute vacuum reaches a radius of $8.7713 \times 10^{8}$ meters, the space inside the sphere will be at its highest level of instability, the only way for it to achieve equilibrium is to collapse inward, creating the first particle.

Universe at zero second, infinity spatial dimensions of absolute vacuum existed. Therefore, unlimited numbers of particles were created simultaneously everywhere.

External Vacuum resistant $=3.2857602 * 10^{\wedge} 15 *$ mass, this equation can be used whenever there is a release in the External vacuum resistant or energy as a result of Internal vacuum resistant conversion.

\section{Acknowledgments}

I would like to express my gratitude to Salahdin university and specifically (Dr Ameer)- Physics department for providing comments and feedback about the current study.

\section{REFERENCES}

1. Adams, F.C. and G.J.R.o.M.P. Laughlin, A dying universe: the long-term fate and evolutionof astrophysical objects. 1997. 69(2): p. 337.

2. La, D. and P.J.J.P.R.L. Steinhardt, Extended inflationary cosmology. 1989. 62(4): p. 376. 
3. Cai, Y.-F.J.S.C.P., Mechanics and Astronomy, Exploring bouncing cosmologies with cosmological surveys. 2014. 57(8): p. 1414-1430.

4. Alpher, R.A., H. Bethe, and G.J.P.R. Gamow, The origin of chemical elements. 1948. 73(7): p. 803.

5. Craig, William Lane, the Tenseless Theory of Time, Springer, 2010.

6. Sirdy, S. (2020, December 31). The Role of Elementary Dimensions in the Creation of the Source of Elementary Particles. UKH Journal of Science and Engineering, 4(2), 69-83. https://doi.org/https://doi.org/10.25079/ukhjse.v4n2y2020.pp69-83

7. Oppenheim, Alan; Willsky, Alan (1997). Signals and Systems (second ed.). Prentice Hall.

8. Pooley, D.; Kumar, P.; Wheeler, J. C.; Grossan, B. (2018-05-31). "GW170817 Most Likely Made a Black Hole". The Astrophysical Journal. 859 (2): L23. arXiv:1712.03240. Bibcode:2018 ApJ...859L..23P. doi:10.3847/2041-8213/aac3d6.

9. Conn, Robert W.. "Nuclear fusion". Encyclopedia Britannica, 5 Jul. 2019, https://www.britannica.com/science/nuclear-fusion. Accessed 23 August 2021. 